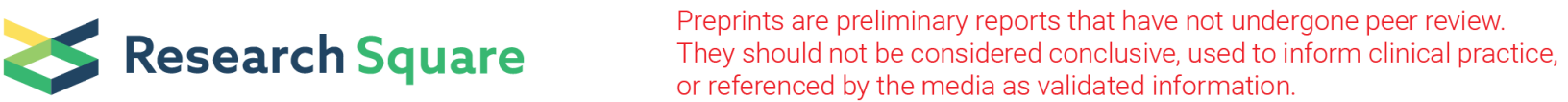 \\ Wideband Spectrum Sensing Based on Advanced Sub-Nyquist Sampling Structure
}

\section{Xue Wang}

Harbin University of Science and Technology https://orcid.org/0000-0002-6824-3223

\section{Qian Chen}

Harbin University of Science and Technology

Min Jia ( $\sim$ jiamin@hit.edu.cn)

Harbin Institute of Technology

Xuemai Gu

Harbin Institute of Technology

\section{Research Article}

Keywords: Wideband spectrum sensing, modulated wideband converter, sub-Nyquist sampling, correct support recovery, blind spectrum sensing

Posted Date: December 10th, 2021

DOI: https://doi.org/10.21203/rs.3.rs-1141719/v1

License: (c) (1) This work is licensed under a Creative Commons Attribution 4.0 International License. Read Full License 


\title{
Wideband spectrum sensing based on advanced sub-Nyquist sampling structure
}

\author{
Xue Wang ${ }^{1}$, Qian Chen ${ }^{1}$, Min Jia ${ }^{2 *}$ and Xuemai Gu${ }^{2}$
}

\footnotetext{
${ }^{*}$ Correspondence:

jiamin@hit.edu.cn

${ }^{2}$ Harbin Institute of Technology,

No.2 Yikuang Street, Harbin,

China

Full list of author information is

available at the end of the article
}

\begin{abstract}
As the bandwidth increases, the high-speed sampling rate becomes the bottleneck for the development of wideband spectrum sensing. Wideband spectrum sensing with sub-Nyquist sampling attracts more attention and modulated wideband converter (MWC) is an attractive sub-Nyquist sampling system. For the purpose of breaking the system structure limit, an advanced sub-Nyquist sampling framework is proposed to simplify the MWC system structure, adopting the single sampling channel structure with a frequency shifting module to acquire the sub-Nyquist sampling values. In order to recover the signal support information, the sensing matrix must be built according to the only one mixing function. Most existing support recovery methods rely on some prior knowledge about the spectrum sparsity, which is difficult to acquire in practical electromagnetic environment. To address this problem, we propose an adaptive residual energy detection algorithm (ARED), which bypasses the need for the above-mentioned prior knowledge. Simulation results show that, without requiring the aforementioned prior knowledge, the ARED algorithm, which is based on the advanced sub-Nyquist sampling framework, has the similar performance as MWC and even higher than MWC in some cases.
\end{abstract}

Keywords: Wideband spectrum sensing; modulated wideband converter; sub-Nyquist sampling; correct support recovery; blind spectrum sensing 
services $[1,2,3]$. Wideband spectrum sensing (WSS) has been widely recognized as an effective means to deal with the increasing demand for broadband access and the scarcity of available spectrum [4]. However, the increasing bandwidth brings a great challenge to the implementation of conventional WSS techniques, thus sub-Nyquist wideband spectrum sensing attracts significant attentions [5].

Based on the compressed sensing (CS) theory, several sub-Nyquist sampling systems have been introduced, such as an analog to information converter (AIC) [6], a multicoset sampling (MCS) [7] and a modulated wideband converter (MWC) [8]. AIC-based WSS can handle discrete multitone signals, i.e. sinusoids with sparse frequencies. When signals have a certain bandwidth, AIC system is no longer applicable, but MCS and MWC still work. These two systems belong to multi-channel structure. MCS is difficult to implement by hardware, because an accurate time delay is strictly required for each channel. By contrast, a prototype of MWC has been implemented. Though its precision is lower than that of MCS at the same sampling rate, MWC is still an attractive alternative.

In terms of signal support set reconstruction, compressed-aware reconstruction algorithms are borrowed, such as orthogonal matching pursuit (OMP), regularized OMP, stagewise OMP, subspace pursuit and compressive sampling MP, which are proposed to solve the Single Measurement Vector (SMV) problem and cannot be directly used to solve the multiband signal reconstruction problem. However, the reconstruction of multiband signals can be transformed into the Multiple Measurement Vectors (MMV) problem. To solve the reconstruction problem of joint sparse signals, five greedy algorithms designed for SMV sparse estimation can be extended to the MMV problem. In addition, many MWC-based signal improvement algorithms are proposed, such as the iterative support detection method [9], and the sparse Bayesian algorithm [10]. Although the MMV class algorithms require fewer samples than the SMV class algorithms to achieve the same signal reconstruction accuracy, the MMV class algorithms [11, 12, 13], like the SMV class algorithms, also rely on the a priori information of signal sparsity, which is extremely difficult to be acquired in the actual complex electromagnetic environment.

In this paper, an advanced sampling framework (ADS) is proposed to achieve subNyquist sampling for multiband signals. Compared to traditional MWC, a prime difference is that advanced sampling framework adopts single-channel structure. In 
order to collect enough sub-Nyquist samples, a frequency shifting module is added. The proposed structure mainly solves the following two problems. First, it can flexibly take control of the number of sub-Nyquist samples. The key point of MWC is to recover the signal supports by exploiting the CS recovery algorithms. However, the CS theory has strict requirements on the observation times, namely the number of sampling channels in MWC. As the number of sub-bands increases, sampling channels must be added to acquire enough samples. Once MWC is implemented in hardware, the number of sampling channels is fixed. In other words, the ability to process multiband signals is limited. In actual scenario, there is no priority knowledge of the number of sub-bands, so it will be difficult in putting MWC in practical applications. This has motivated interest in the proposed structure to overcome the limitations of traditional MWC. Second, the input signal to MWC is mixed with a high-speed pseudorandom chip sequence. This operation puts the pressure on hardware implementation and advanced structure can greatly reduce this operation. Based on single-channel structure, wideband spectrum sensing can be realized to monitor high dynamic electromagnetic spectrum and detect non-cooperative signals.

The reminder of the paper is organized as follows. In Section 2, the system model of MWC system is first briefly reviewed. Then, the single-channel sub-Nyquist sampling structure is proposed in Section 3. Finally, simulation results are presented in Section 4 for demonstrating the performance of the proposed approach.

\section{System model}

MWC allows the RF signal to be sampled using existing ADCs, reducing the limitations on hardware. Compared to multi-coset sampling, there is no requirement for strict time synchronization. Meanwhile, the appearance of prototypes makes MWC draw more attention.

\subsection{MWC architecture}

MWC uses a fixed analog sampling front end, which contains $m$ parallel sampling channels, each consisting of a mixer, low-pass filter and sampler, as shown in Fig.1. 


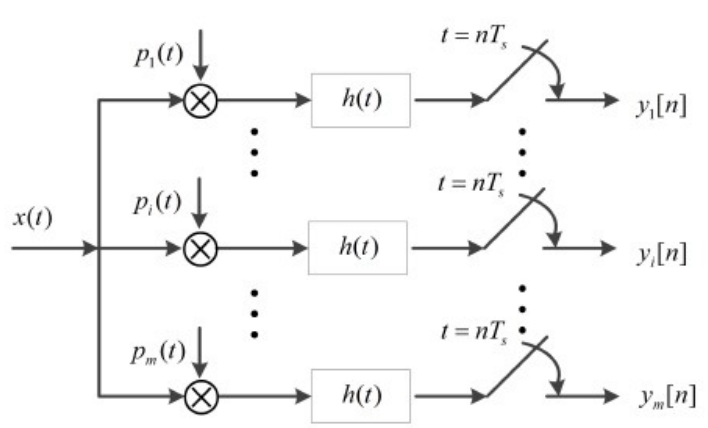

Figure $1 \mathrm{MWC}$ analog sampling front end.

The RF signal $x(t)$ is an analog multiband signal with a bandwidth range $F=$ $[-1 / 2 T, 1 / 2 T)$, and its Fourier transform is as follows.

$$
X(f)=\int_{-\infty}^{\infty} x(t) e^{-j 2 \pi f t} d t
$$

Once the multiband signal $x(t)$ enters MWC, $m$ sampling channels simultaneously perform the same processing process on the RF signal. First, the RF signal $x(t)$ is modulated by the mixing function through the mixer. The mixing function $p_{i}(t)$ is $T_{p}$-periodic, containing $M$ code slices in each period. Each code slice varies between \pm 1 , as shown in Fig.2. The periodic pseudo-random sequence is chosen as the mixing function.

$$
p_{i}(t)=\alpha_{i k}, k \frac{T_{p}}{M} \leq t \leq(k+1) \frac{T_{p}}{M}, 0 \leq k \leq M-1
$$

With $\alpha_{i k} \in\{+1,-1\}$ and $p_{i}\left(t+n T_{p}\right)=p_{i}(t)$ for any $n \in \mathrm{Z}$.

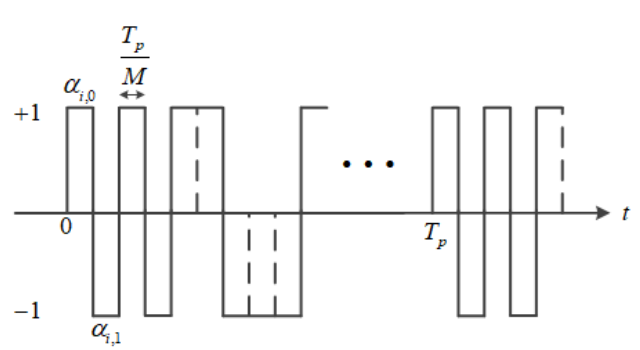

Figure 2 The mixing function $p_{i}(t)$. 
Considering the $i$ th branch, since $p_{i}(t)$ is a periodic signal, its Fourier expansion form is

$$
p_{i}(t)=\sum_{l=-\infty}^{\infty} c_{i l} e^{j \frac{2 \pi}{T_{p}} l t}
$$

Where,

$$
c_{i l}=\frac{1}{T_{p}} \int_{0}^{T_{p}} p_{i}(t) e^{-j \frac{2 \pi}{T_{p}} l t} \mathrm{~d} t
$$

The multiband signal $x(t)$ is multiplied with $p_{i}(t)$ to obtain the modulated signal $\tilde{x}_{i}(t)=x(t) p_{i}(t)$, whose Fourier transform is

$$
\begin{aligned}
\tilde{X}_{i}(f) & =\int_{-\infty}^{\infty} \tilde{x}_{i}(t) e^{-j 2 \pi f t} \mathrm{~d} t \\
& =\int_{-\infty}^{\infty} x(t)\left(\sum_{l=-\infty}^{\infty} c_{i l} e^{j \frac{2 \pi}{T_{p}} l t}\right) e^{-j 2 \pi f t} \mathrm{~d} t \\
& =\sum_{l=-\infty}^{\infty} c_{i l} X\left(f-l f_{p}\right)
\end{aligned}
$$

$X(f)$ is the Fourier transform of $x(t)$ and $f_{p}=1 / T_{p}$. Through the mixer, the modulated signal spectrum $\tilde{X}_{i}(f)$ generates the spectrum aliasing effect, which manifests itself as a linear weighting combination of $f_{p}$-shift copies of $X(f)$ and the weighting factor is the Fourier coefficient $c_{i l}$. The operation is similar to the spread spectrum, the multiband signal spectrum will be extended, so that the spectrum spans full spectrum, at this time extended to the low frequency part. Notice that the $m$ parallel channels differ only in the pseudo-random sequence chosen for the mixing function, the other operations are identical.

Subsequently, the modulated signal is filtered using a low-pass filter to retain some information in the signal baseband, and the filtered signal is sampled at low speed to obtain the sampling signal $y_{i}[n]$ of each branch. The cutoff frequency of the lowpass filter is $1 / 2 T_{s}$ as shown in Fig.3 and the sampling rate is $1 / T_{s}$. Suppose the low-pass filter frequency response be $\mathbf{H}_{L P F}(f)$, the sampled value $y_{i}[n]$ contains only the frequency component $f \in F$.

The discrete-time Fourier transform (DTFT) of $y_{i}[n]$ is

$$
\begin{aligned}
Y_{i}\left(e^{j 2 \pi f T_{s}}\right) & =\sum_{n=-\infty}^{\infty} y_{i}[n] e^{-j 2 \pi f n T_{s}} \\
= & \sum_{l=-L_{0}}^{+L_{0}} c_{i l} X\left(f-l f_{p}\right), \quad f \in F
\end{aligned}
$$




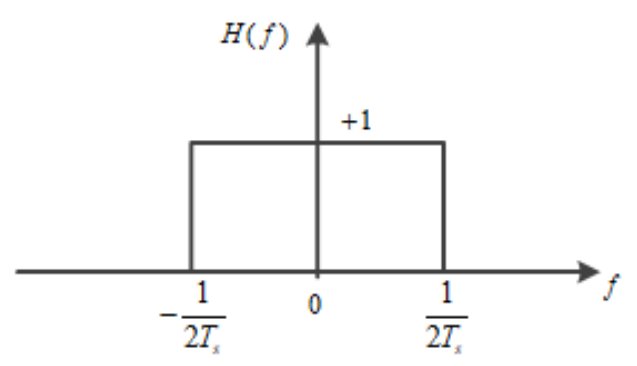

Figure 3 Frequency response of low pass filter.

Where $L_{0}$ denotes all possible non-zero terms of $X(f)$ in the positive frequency part of the signal bandwidth $F$,

$$
-\frac{f_{s}}{2}+\left(L_{0}+1\right) f_{p} \geq \frac{f_{N Y Q}}{2} \rightarrow L_{0}=\left\lceil\frac{f_{N Y Q}+f_{s}}{2 f_{p}}\right\rceil-1
$$

Equation 6 relates the spectrum of the sampled sequence $y_{i}[n]$ to the spectrum of the unknown signal $X(f)$ in the frequency domain, which constructs a classical reconstruction formula similar to the compressed sensing. Rewrite it as matrix form.

$$
\mathbf{Y}(f)=\mathbf{A} \mathbf{z}(f), \quad f \in F
$$

The $i$ th element of a $m \times 1$ vector $\mathbf{Y}(f)$ is $Y_{i}\left(e^{j 2 \pi f T_{s}}\right) . \mathbf{z}(f)$ is $L \times 1$ with $L=2 L_{0}+1$,

$$
\mathbf{z}(f)=X\left(f+\left(i-L_{0}-1\right) f_{p}\right), f \in F, 1 \leq i \leq L
$$

Matrix $\mathbf{A}$ is $m \times L$ with the elements $\mathbf{A}_{i l}=c_{i,-l}=c_{i l}^{*},-L_{0} \leq l \leq L_{0}$.

After the mixing function $p_{i}(t)$, the multiband signal is periodically extended in the frequency domain. Each sub-band signal in the baseband part is obtained after passing the low-pass filter, and then the sub-band signals are mixed together. The data stream $y[n]$ is sampled by low-speed rate. The signal support set information is acquired by compressed sensing theory, and separate the mixed sub-band signals.

\subsection{Discussions}

MWC has drawn more attention among many wideband signal sub-Nyquist sampling systems. It can process multiband signals at sub-Nyquist rates without the prior of the exact location of each sub-band, but has application limitations. The 
two core components of MWC: the front-end sub-Nyquist sampling structure and subsequent signal processing algorithms, which both limit the use of MWC.

Front-end sub-Nyquist sampling structure employs multi-channel parallel structure. When implemented in hardware, the number of sampling channels is then determined and cannot be changed. The number of sampling channels directly affects the accuracy of the subsequent signal support set recovery algorithm. Taking the existing signal support set algorithm as an example, two prerequisites are required to recover the support set accurately, a priori information on the number of signals required and the number of sampling channels to match the number of sub-bands. In a complex electromagnetic environment, the number of signals is not known in advance and is also constantly changing over time. Such uncertainty directly limits the application of MWC. Another perspective is that when the number of sampling channels is fixed, the upper limit of the number of signals it can detect will also be determined.

As above, the main contributions of this paper are as follows. On the one hand, design a single-channel advanced structure, which can flexibly select the number of sampling values according to the number of signals changing. At the same time, it can ensure the effective operation of the support set recovery algorithm, so that the sampling process is channel selection adaptive. On the other hand, the signal support set blind recovery algorithm, called adaptive residual energy detection algorithm, is proposed to eliminate the priori information about the signal number, which adapts to dynamic electromagnetic environment.

\section{Single-channel advanced sampling method}

\subsection{Single-channel Sub-Nyquist sampling Structure}

The aliasing effect of the modulated signal spectrum due to the period extension is the basis for the implementation of sub-Nyquist sampling in MWC systems. The spectrum of the modulated signal $\tilde{X}_{i}(f)$ appears as a weighted linear combination of $f_{p}$-shift copies of $X(f)$. Assuming that the spectrum range is divided into several intervals by $f_{p}$ length, every $f_{p}$-length interval contains the weighted spectrum information from each sub-band, which is considered as the drivers for single-channel sub-Nyquist sampling structure. 
Using a single-channel equivalent to a conventional MWC system, the core idea is to combine the frequency-shifting properties of the Fourier transform. Taking full advantage of the spectral panning feature of single-channel signals, the mixed signals in each region of $f_{p}$ length are considered as the signals acquired by one sampling channel. The period-weighted expansion of the spectrum of one sampling channel is extracted, and the signal in each region is equivalent to the signal obtained from multiple other sampling channels. Therefore, the single channel needs to add a frequency shift module between the mixer and the low-pass filter, and the modulated signal will be frequency shifted and passed through the low-pass filter in turn. Several linearly weighted signals in the $f_{p}$-length region are reserved according to the actual requirements to equate to the road sampling channels of a conventional MWC system.

The processed signal $\hat{x}(t)=\tilde{x}(t) \cdot e^{-j 2 \pi\left(a \cdot f_{p}\right) t}$ produces a frequency shift effect in the frequency domain.

$$
\begin{aligned}
\hat{X}(f) & =\int_{-\infty}^{\infty} \hat{x}(t) \cdot e^{-j 2 \pi f t} \mathrm{~d} t \\
& =\int_{-\infty}^{\infty} \tilde{x}(t) \cdot e^{-j 2 \pi\left(a \cdot f_{p}\right) t} \cdot e^{-j 2 \pi f t} \mathrm{~d} t \\
& =\tilde{X}\left(f+a \cdot f_{p}\right)
\end{aligned}
$$

Where $\tilde{X}(f)$ is the spectrum of the modulated signal and $a \in \mathbf{N}+$. Different channels are equated by selecting different values of $a$.

The spectrum of the modulated signal $\tilde{x}(t)$, after different frequency shift operations, is used as the input signal $\hat{x}(t)$ for the low-pass filter.

$$
\hat{x}(t)=\tilde{x}(t) \cdot e^{-j 2 \pi\left(a \cdot f_{p}\right) t}=x(t) p(t) \cdot e^{-j 2 \pi\left(a \cdot f_{p}\right) t}
$$

Its Fourier expansion takes the form

$$
\begin{aligned}
\hat{X}(f) & =\int_{-\infty}^{\infty} \hat{x}(t) e^{-j 2 \pi f t} \mathrm{~d} t \\
& =\int_{-\infty}^{\infty} x(t)\left(\sum_{l=-\infty}^{\infty} c_{l} e^{j \frac{2 \pi}{T_{p}} l t}\right) e^{-j 2 \pi\left(a \cdot f_{p}\right) t} \cdot e^{-j 2 \pi f t} \mathrm{~d} t \\
& =\sum_{l=-\infty}^{\infty} c_{l} X\left(f-(l-a) f_{p}\right)
\end{aligned}
$$

The filtered signal $Y(f)=\hat{X}(f) \cdot \mathbf{H}_{L P F}(f)$ retains only the spectrum located in the baseband portion, which is a linear combination of each sub-band with different weighting factors. By setting different values of $a$ to obtain sub-Nyquist sampling 
values sufficient, it can satisfy the reconstruction of signal support information. These sample values can be equivalently considered as coming from different sample channels. In this paper, the relationship between sampling channel number $i$ and parameter $a$ is specified as follows.

$$
i=a+1
$$

Where $i$ and $a$ take the values of $i=[1,2, \cdots, m]$ and $a=[0,1, \cdots, m-1]$, respectively. $m$ is the equivalent number of sampling channels, Sampling channel $i=1$ is the sub-Nyquist sampled value obtained when no frequency shift $(a=0)$ occurs, while sampling channel $i \geq 2$ is the modulated signal spectrum obtained by sequentially shifting $f_{p}$ distances to the left.

\subsection{Sensing matrix design}

In the MWC system, the observation matrix $\mathbf{A}$ is constructed based on the Fourier series $c_{i l}$ of the different mixing functions $p_{i}(t)$. Matrix $\mathbf{A}$ is $m \times L$, with the elements $\mathbf{A}_{i l}=c_{i,-l}=c_{i l}^{*},-L_{0} \leq l \leq L_{0}$, where $m$ denotes the number of sampling channels and $L$ denotes the number of regions of $f_{p}$ length divided in the spectrum sensing range. The observation matrix $\mathbf{A}$ of MWC is composed of $m$ mixing functions $p_{i}(t)$ and the form is as follows.

$$
\mathbf{A}=\left[\begin{array}{ccccccc}
c_{1,-L_{0}} & c_{1,-L_{0}+1} & \cdots & c_{1,0} & \cdots & c_{1, L_{0}-1} & c_{1, L_{0}} \\
c_{2,-L_{0}} & c_{2,-L_{0}+1} & \cdots & c_{2,0} & \cdots & c_{2, L_{0}-1} & c_{2, L_{0}} \\
\vdots & \vdots & \ddots & \vdots & \ddots & \vdots & \vdots \\
c_{m,-L_{0}} & c_{m,-L_{0}+1} & \cdots & c_{m, 0} & \cdots & c_{m, L_{0}-1} & c_{m, L_{0}}
\end{array}\right]
$$

Each column of matrix A corresponds to a region of $f_{p}$ length in the sensed spectrum range, and the weighting factor of each sub-band located in the baseband part is related to the location of the sub-band. The weighting factor of each subband period extension is known by equation 4 .

Matrix A has conjugate symmetry. From the amplitude point of view, the leftward and rightward periodic extension spectrum in the baseband part has the same amplitude variation. 
In the single-channel structure, the frequency-shifted modulated signal spectrum is as in equation 12 . Let $l^{\prime}=l-a$ and substitute $l=l^{\prime}+a$ into equation 12 ,

$$
\hat{X}(f)=\sum_{l^{\prime}=-\infty}^{\infty} c_{l^{\prime}+a} X\left(f-l^{\prime} f_{p}\right)=\sum_{l=-\infty}^{\infty} c_{l+a} X\left(f-l f_{p}\right)
$$

Comparing with equation 12, the weighting factor of the spectrum is $c_{l+a}$.

In the single-channel structure, there is only one mixing function, and the observation matrix $\mathbf{A}$ is constructed using the Fourier series of one mixing function corresponding to the frequency shift operation. The weighting factor for each sampling channel corresponds to one row of matrix $\mathbf{A}$. Therefore, $\mathbf{A}$ can be obtained by a row-by-row translation in a single channel structure.

$$
c_{i, l}=c_{l+a}=c_{l+(i-1)}, \quad 1 \leq l \leq L_{0}-(i-1)
$$

where $i=a+1$. At this point, the elements in matrix A no longer satisfy the conjugate symmetry relationship. As long as the constructed matrix $\mathbf{A}$ is consistent with the sampled values after the translation operation, it will not affect the subsequent reconstruction of the sub-band support information.

In the single-channel structure, the Fourier series $c_{l}$ of the mixing function is used as the first row of matrix $\mathbf{A}$, and the remaining rows are generated by shifting the elements of the first row according to equation 16. After the translation, there are free positions in $\mathbf{A}$ that need to be filled to complete the construction of $\mathbf{A}$. There can be 2 ways to fill the idle position: shift method, which uses the complementary zero method to fill the idle position left after translation, and cyclic shift method, which uses the cyclic shift method to fill the idle position left after translation.

\subsection{Sampling structure improvement}

The frequency shift module is the most central device of the sub-Nyquist sampling system based on the single-channel structure, which is not only the key to ensure the proper operation of the system, but also can increase the flexibility of sampling value acquisition. Two basic structures are given in this section, i.e., parallel structure and series structure, as shown in Fig.4 and Fig.5.

In both structures, a low-pass filter is used to obtain multiple sub-Nyquist sampled values through the control of the timing. The difference between the two structures 


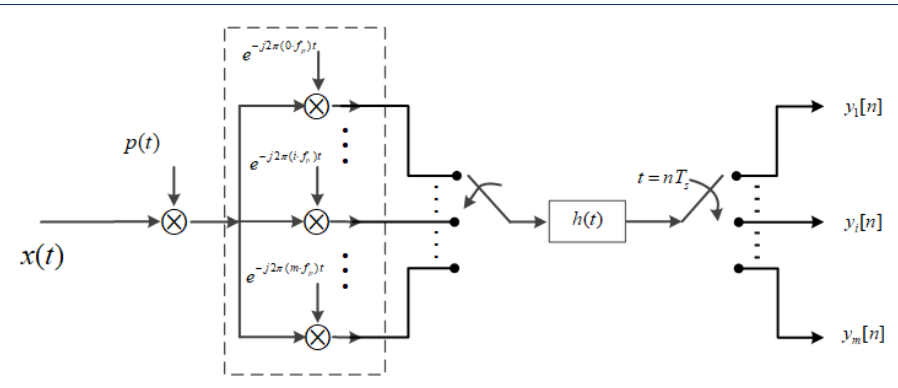

Figure 4 Frequency shifting module with parallel structure.

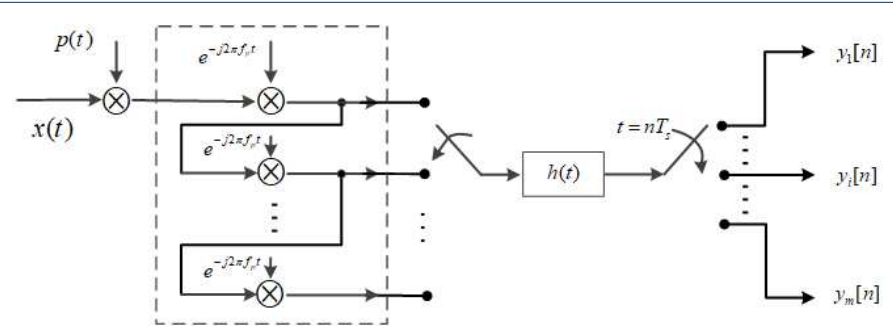

Figure 5 Frequency shifting module with serial structure.

is that the parallel structure can acquire the baseband spectrum without frequency shifting as the sampled signal, while the sampled signal acquired in the series structure is the frequency shifted signal spectrum. The parallel structure uses different frequency shifters, while the series structure can use the same frequency shifters.

Wideband spectrum sensing has high requirements for timeliness. When the above-mentioned single channel advanced sampling structure is adopted, the efficiency of the frequency shift module will directly affect the working time and efficiency of the whole system and it becomes the key to system performance improvement. Considering that the low-pass filter bandwidth in the system matches the sub-band bandwidth of the multiband signal, the sampling rate can be appropriately increased in order to shorten the signal processing time delay caused by frequency shift. In addition, the sampling process is flexibly controlled according to actual needs, making it more adaptable to application scenarios where the number of signals is unknown and constantly changing.

The mixing function used in the mixer can be a pseudo-random sequence, which values switch between 1 and -1 . For better hardware implementation, the mixing function can be improved to ensure that the total number of code pieces remains the same during the period. 
First, the value of each code slice of the modulation function is selected as 0 or 1 . The mixer can be implemented through the control of the high-frequency switch. The opening of the high-frequency switch corresponds to the code piece whose mixing function takes the value of 1 to ensure the normal passage of the signal, while the closing of the switch corresponds to the code piece whose mixing function takes the value of 0 to achieve the modulation of the input multiband signal. Secondly, in order to reduce the opening and closing frequency of the high frequency switch, the mixing function structure can be changed so that a number of successive adjacent elements take the same value, thus reducing the opening and closing frequency of the switch exponentially and reducing the difficulty of hardware implementation.

In the improved mixing function, it is required that the number of code pieces remains the same $M$ for a cycle $T_{p}$. As the number of code slices with the same value increases, it makes the mixing function less random and will affect the reconstruction results of the signal support set to some extent.

\subsection{The proposed ARED algorithm}

In this section, the proposed adaptive residual energy detection algorithm (ARED) is similar to other algorithms for support set solution based on the greedy compressed sensing algorithm. The processing of the proposed ARED algorithm includes matching the residuals with the observation matrix, and then finding the most relevant columns to obtain the corresponding support set information, updating the residuals again, and performing circular matching until the complete support set information is obtained. In this algorithm, the iteration termination condition is no longer the sparsity of the signal, but the difference of two mean square errors is chosen. It can effectively solve the difficult problem that the signal sparsity cannot be known in advance, and solve the reconstruction problem of signal support set with arbitrary sparsity more flexibly. The pseudo-code of the proposed algorithm is shown in Algorithm 1.

In step 1, at the beginning of each iteration, the correlation between the residuals and each column of the observation matrix is solved to find the best matching column, whose corresponding column number $Z^{\kappa}$ is a support set information. Con- 
sidering that the spectrum of the real signal has conjugate symmetry, the signal support set $\tilde{S}^{\kappa}$ is thus updated as $Z^{\kappa}$ and $L+1-Z^{\kappa}$.

In step 2, the multiband signal after this iteration is obtained through the pseudoinverse matrix $\mathbf{A}_{\tilde{S}^{\kappa}}^{\dagger}$ and the residuals are updated.

In step 3 , the observation matrix $\mathbf{A}^{\kappa}$ is updated with the diagonal correction matrix so that the energy of each row of the observation matrix is normalized to 1.

The details of the algorithm are shown in Algorithm 1. Compared with the tra-

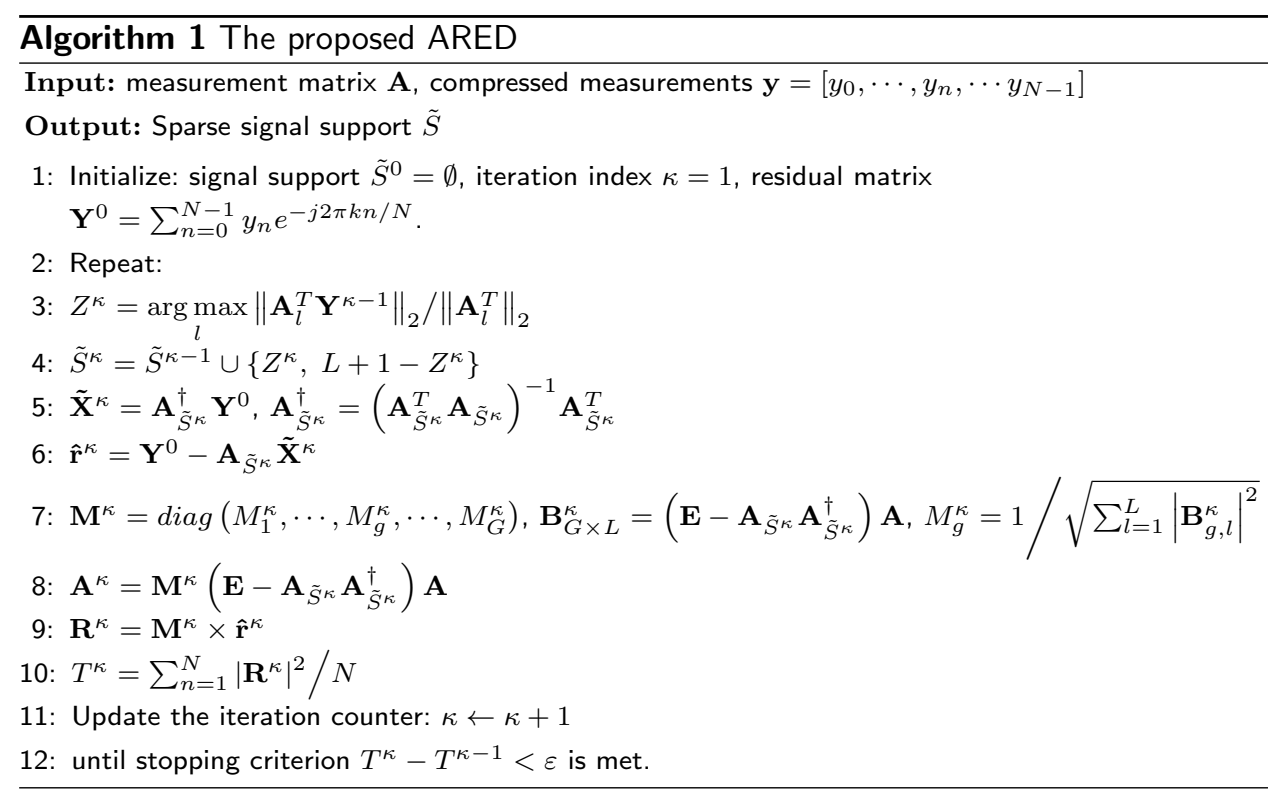

ditional MWC system signal support set recovery algorithm, the proposed ARED algorithm no longer requires the a priori information of signal sparsity and uses the residual energy detection result as the iterative termination condition to achieve blind detection of the signal.

\section{Simulation result}

In this section, we will verify the above analyses and compare the performance along with the original multi-channel MWC system. In our experiment, the QPSK signal is selected as the sub-band signal,

$$
x(t)=\sum_{i=1}^{N_{/ 2}} \sqrt{\frac{2 E_{s i}}{T_{s i}}}\left\{\begin{array}{l}
\sum_{n} I[n] s\left(t-n T_{s}\right) \cos \left(2 \pi f_{i} t\right) \\
+\sum_{n} Q[n] s\left(t-n T_{s}\right) \sin \left(2 \pi f_{i} t\right)
\end{array}\right\}
$$

Where $N$ is the number of sub-band signal, $T_{s i}=4 \times 10^{-2} \mu s$ is symbol duration and symbol energy $E_{s i}$ is random selection. The in-phase and quadrature bit streams 
are $I[n], Q[n]$ and the generated bit streams uniformly at random with $n=150$ symbols. $s(t)=\operatorname{sinc}\left(t / T_{s}\right)$ is the pulse shaping. The carriers $f_{i}$ are chosen uniformly at random over a wideband range with $f_{N y q}=5 \mathrm{GHz}$. The multiband signal is composed of three QPSK signals with $S N R=10 \mathrm{~dB}$. Matrix $\mathbf{A}$ is obtained by cyclic shift method and use the proposed ARED algorithms to recover the frequency supports. The signal processing results are shown in Fig.6.
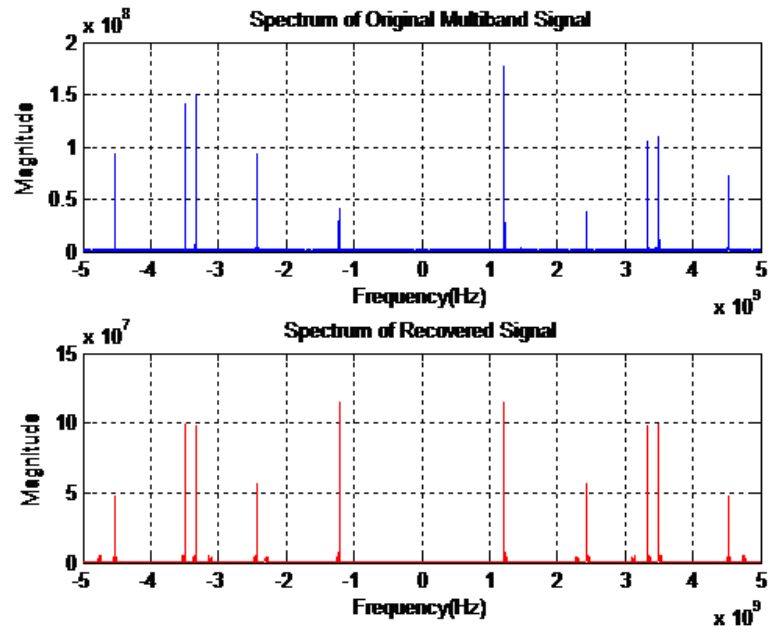

Figure 6 Spectrum comparison diagram of signal reconstruction in ADS.

A successful reconstruction of the signal support set is defined when the support set of the reconstructed signal $S_{R}$ contains the support set of the original signal $S_{O}$,

$$
S_{R} \supseteq S_{O}
$$

$S_{R}=S_{O}$ means the exact reconstruction of the support set. Due to the influence of noise, there will be support set false alarm situation, if completely eliminate the false alarm situation need to use other signal processing methods, not in the scope of this paper. Considering that the primary principle of shared spectrum is that the work of its own system does not affect the normal work of other systems, the support set reconfiguration success with equation 18 defined in this paper meets this requirement. Ensuring that all sub-band locations are accurately located, the provided spectrum access locations do not interfere with the work of other existing systems. 


\subsection{Support set detection performance}

For MWC and ADS systems, the setting of parameters will directly affect the performance of signal support set reconstruction. The three most influential parameters are the average signal-to-noise ratio, the number of sampling channels (number of sub-Nyquist sampled values) and the number of sub-bands (joint sparsity), and the following simulations analyze the effects of these three factors. Since the signal and observation matrices are random, there are inevitable fluctuations in the probability of successful reconstruction of the signal support set, and the following simulation results are obtained by statistics of 1000 Monte Carlo simulations.

The simulation parameters are as follows: the number of sampling channels is taken from 10 to 100 with an interval of 10 , the average SNR is from $-30 \mathrm{~dB}$ to $30 \mathrm{~dB}$ with an interval of 5 . The number of sub-bands is $N=6$.

As can be seen in Fig.7 and Fig.8, the detection rates of both MWC and ADS systems for the signal support set are increasing as the average signal-to-noise ratio increases and the number of undersamples increases. In contrast, the detection rate of the ADS system for the signal support set is higher at low undersampling numbers, while at high undersampling numbers, the detection rates of both systems are similar.

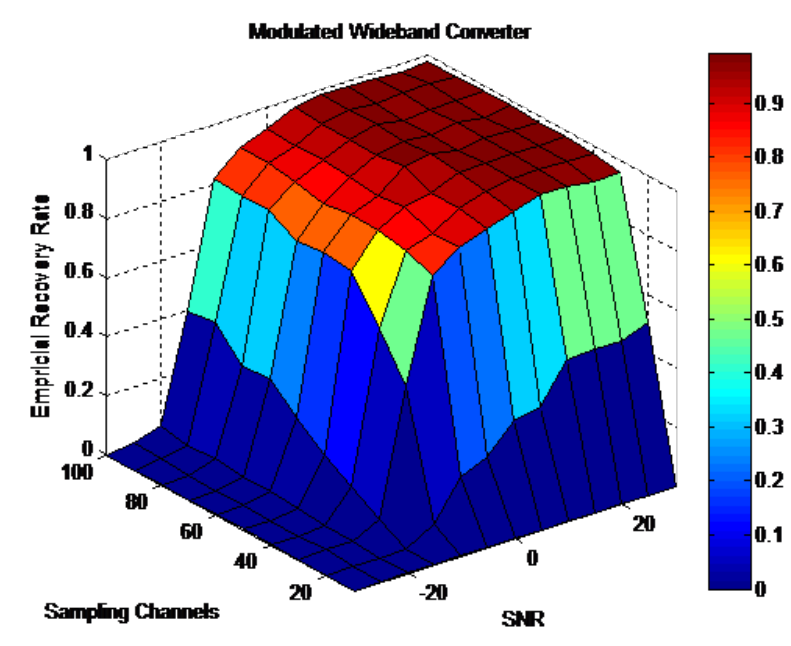

Figure 7 Support set detection performance in MWC.

Specifically, the detection performance of the two systems is compared when the undersampling numbers are taken as 10, 30, 60 and 100, respectively. When the 


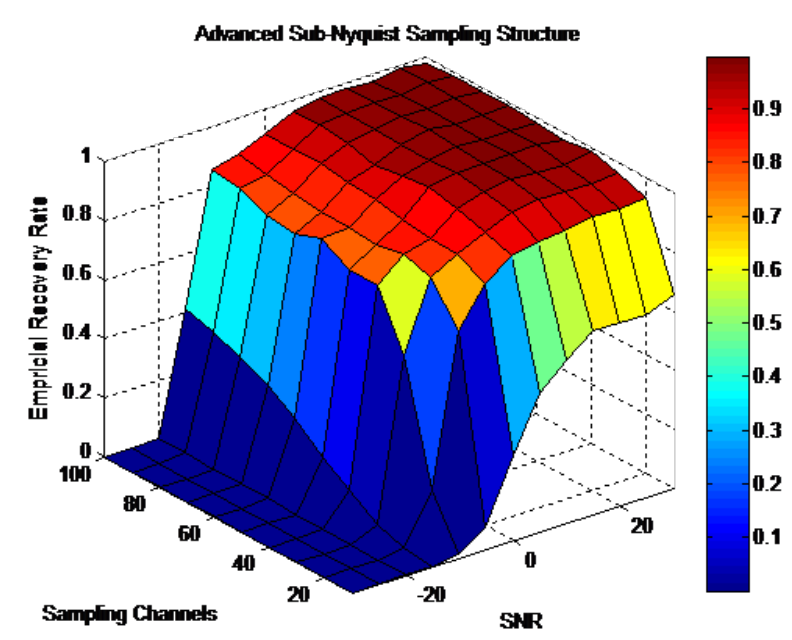

Figure 8 Support set detection performance in ADS.

number of undersamples is greater than 30 , the performance of the two systems does not differ much and both improve with the increase of the average signal-tonoise ratio. Only when the number of undersamples is above 60 , the performance of the system is not improved much simply by increasing the number of undersamples. The number of undersamples directly determines the hardware complexity of the sampling system, especially for MWC systems, the number of undersamples directly affects the number of sampling channels. Therefore, MWC systems will be extremely limited in practical applications. When the number of sampling channels is only 10, the ADS system is improved with the increase of the average signal-to-noise ratio of the signal, but the final undersampling number is too small to achieve better detection efficiency. MWC at this time is unable to complete the normal detection, so the ADS system has a certain detection capacity under the low number of samples, which can be the focus of subsequent research in this scenario.

Fig.9 also shows that ADS outperforms MWC at low undersampling numbers. While both systems have comparable performance with gradually increasing number of samples, as shown in Fig.10

The effects of different numbers of subbands on the detection results are given in Fig.11 and Fig.12 for undersampling numbers of 30 and 60, respectively. It can be seen that the detection performance gradually decreases as the number of subbands increases. And the higher the average signal-to-noise ratio and the higher the num- 


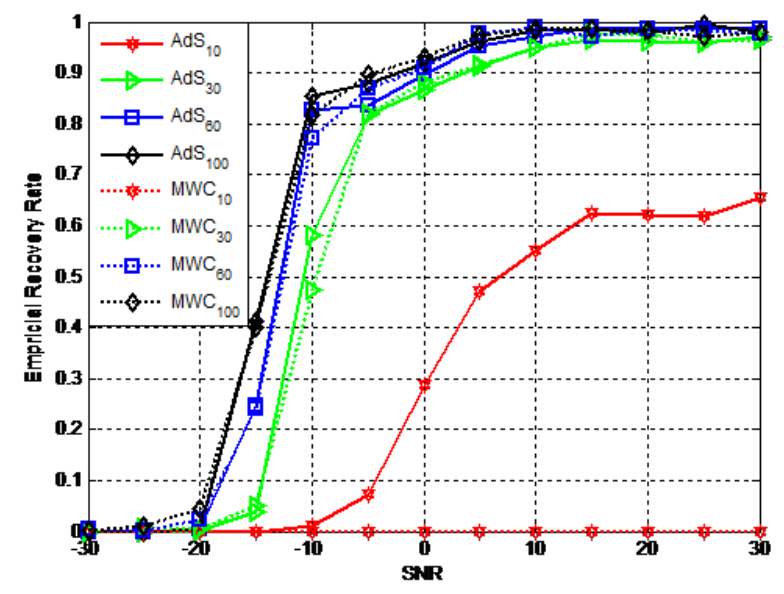

Figure 9 Percentage of correct support recovery, when $m=10,60,100$.

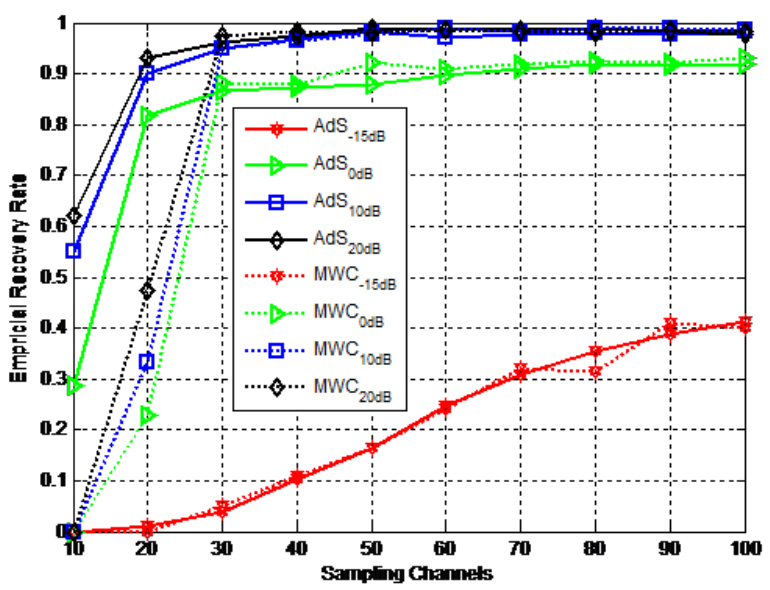

Figure 10 Percentage of correct support recovery, when SNR=-15dB, $0 \mathrm{~dB}, 10 \mathrm{~dB}$ and $20 \mathrm{~dB}$.

ber of samples, the higher the success rate of detection of the signal support set under the same sub-band number condition.

As shown in Fig.11, when the number of samples is 30, the detection performance of the MWC system and the ADS system differs significantly. And when the number of samples is increased to 60 , the detection performance of both systems tends to be similar, as shown in Fig.12. Again, it shows that the ADS system has better detection capability than MWC at low sample number condition. 


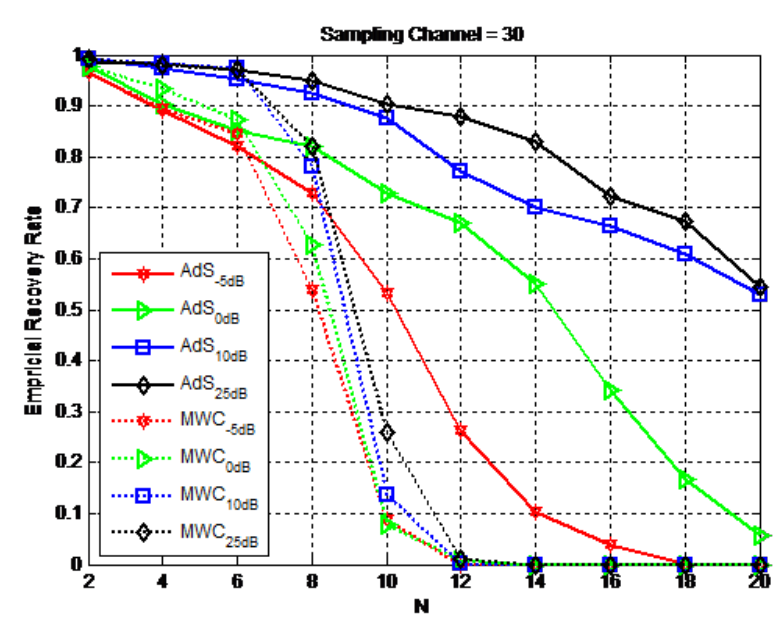

Figure 11 Percentage of correct support recovery, when SNR=-5dB, $0 \mathrm{~dB}, 10 \mathrm{~dB}, 25 \mathrm{~dB}$ and $m=30$.

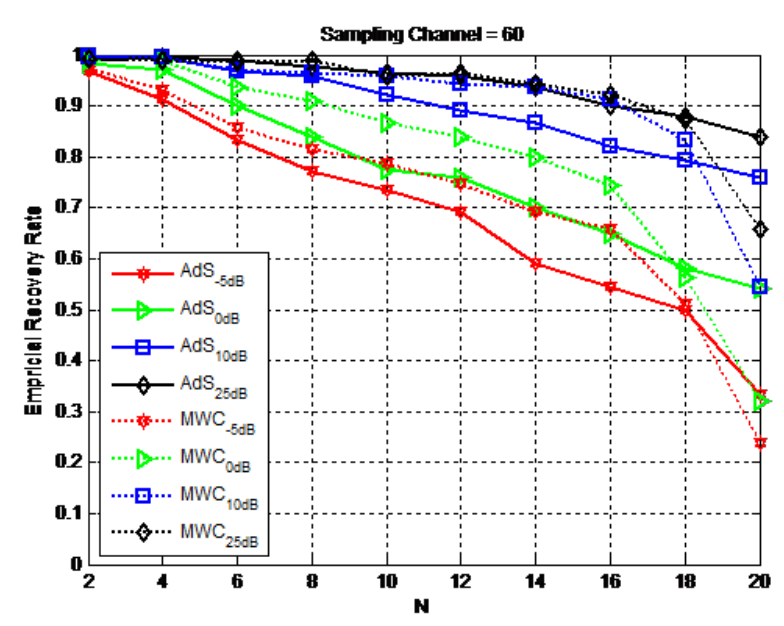

Figure 12 Percentage of correct support recovery, when $S N R=-5 d B, 0 d B, 10 d B, 25 d B$ and $m=60$.

Compared to MWC systems, ADS systems also have the ability to detect multiband signals, and both have similar performance. In particular, ADS is more advantageous when the number of samples is small. MWC only performs better when the number of samples is large enough, and more samples means an increase in the number of sampling channels, which ultimately leads to a significant increase in hardware complexity. In contrast, ADS is more flexible for acquiring sample values and can obtain more samples without increasing hardware complexity. 
Another issue of concern is that in a complex electromagnetic environment, the number of signals that exist simultaneously at a given moment is unpredictable. For MWC systems, once the analog front-end is implemented in hardware, the number of sampling channels will also be determined, and its ability to handle multi-band signals is basically fixed, i.e., the upper limit of the number of sub-bands that can be handled is known. When the number of changing RF signals exceeds the limit, the system will not work. While an increase in the number of sub-bands means that the number of samples must be increased to ensure the effectiveness of signal support set detection, the analog sampling front-end of the ADS system can flexibly cope with different numbers of sub-bands. Therefore, in the complex electromagnetic environment where the signal cannot be known in advance, the ADS system is more valuable for application.

\subsection{Sub-Nyquist sampling structure performance}

The core device of the MWC system is the mixer, which is used to extend all subbands undifferentiated to the baseband by the action of the mixing function, which is filtered by a low-pass filter and then the signal is sampled. Therefore, the mixer is the basis for the MWC to achieve sub-Nyquist sampling and is also the key for the MWC to be able to perform blind processing of RF signals appearing anywhere in the sensing band.

The frequency of each code piece of the mixing function is equal to the Nyquist frequency. Therefore, in order to achieve the modulation effect, the clock frequency controlling the mixing function needs to reach the Nyquist rate, which is a challenge for the hardware implementation process of the sub-Nyquist sampling system. In order to reduce the clock frequency, the mixing functions are designed to be improved, and the performance of the improved four mixing functions in MWC systems and ADS systems is given in this section. The four mixing functions are independent of each other, two consecutive code pieces, four consecutive code pieces, eight consecutive code pieces.

The performance curves of the four modulation functions with different average signal-to-noise ratios for the number of sampling channels of 60 in the MWC system and the ADS system are given in Fig.13 and Fig.14. It can be seen that reducing the clock frequency and extending the duration of the mixing function code slice 
makes the system performance degraded, especially for MWC systems, and in contrast, the ADS system proposed in this paper has better adaptability. The reason is that the MWC system requires randomness among the mixing functions, and when the clock frequency is doubled, the randomness among the mixing functions decreases significantly, and the correlation among the column vectors in the corresponding observation matrix increases, which directly affects the reconstruction of the subsequent signal support set. For the ADS system, the observation matrix is obtained by translation between the rows, and the column vectors are guaranteed to be somewhat uncorrelated, so the effect of clock frequency reduction is not as pronounced as MWC.

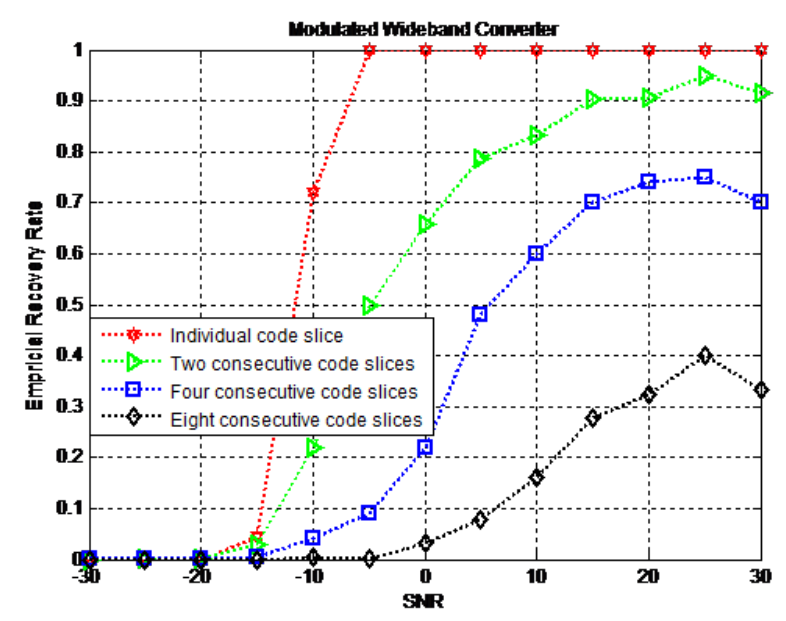

Figure 13 Percentage of correct support recovery in MWC, when $m=60$.

Fig.15 and Fig.16 show the performance curves of the four mixing functions in the MWC system and the ADS system for an average signal-to-noise ratio of $0 \mathrm{~dB}$ and for different numbers of sampling channels. The same conclusion can be drawn that decreasing the clock frequency leads to a decrease in system performance, which has a much greater impact on the MWC system than on the ADS system.

After reducing the clock frequency, the success rate of signal support set reconstruction keeps increasing with the increase of the number of sampling channels in ADS system, while the increase of the number of sampling channels in MWC system has little effect on the system performance improvement. Therefore, the system performance can be improved by increasing the sampling value in the ADS system, 


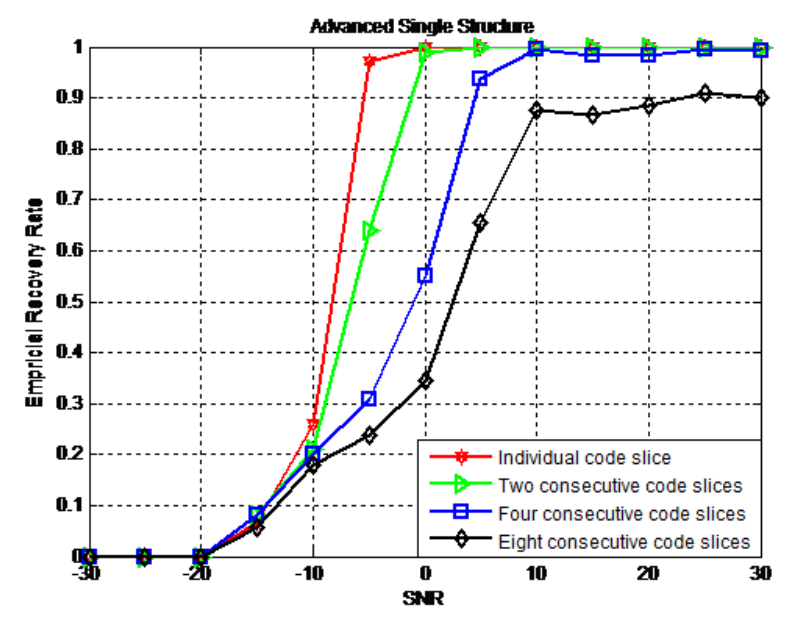

Figure 14 Percentage of correct support recovery in ADS, when $m=60$.

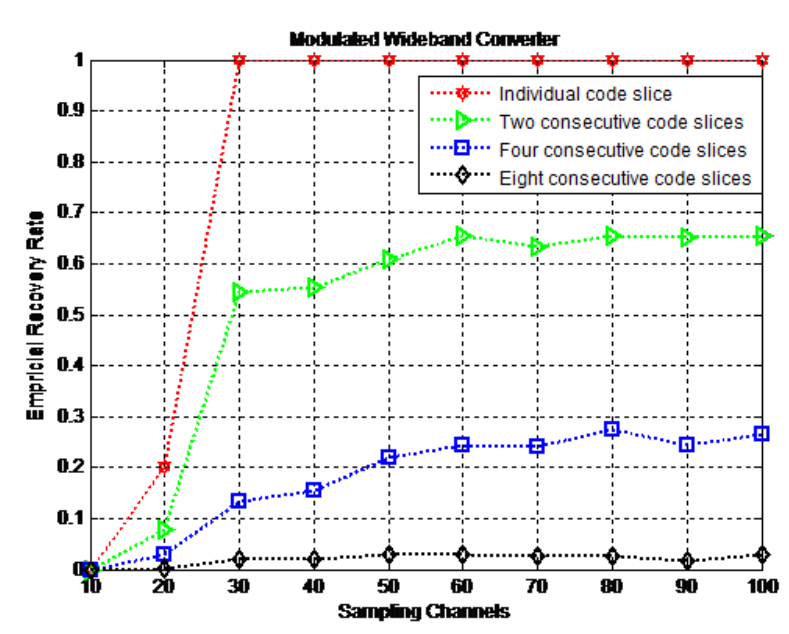

Figure 15 Percentage of correct support recovery in MWC, when SNR=0dB.

and the flexibility in obtaining the sampling value is the structural advantage of the ADS system.

\section{Conclusion}

Face to the issue of spectrum scarcity, wideband spectrum sensing with sub-Nyquist sampling is considered as one of the effective means. In this paper, an advanced sub-Nyquist sampling framework is proposed to simplify the multi-channel MWC system structure. Combined with the frequency shifting properties of the Fourier 


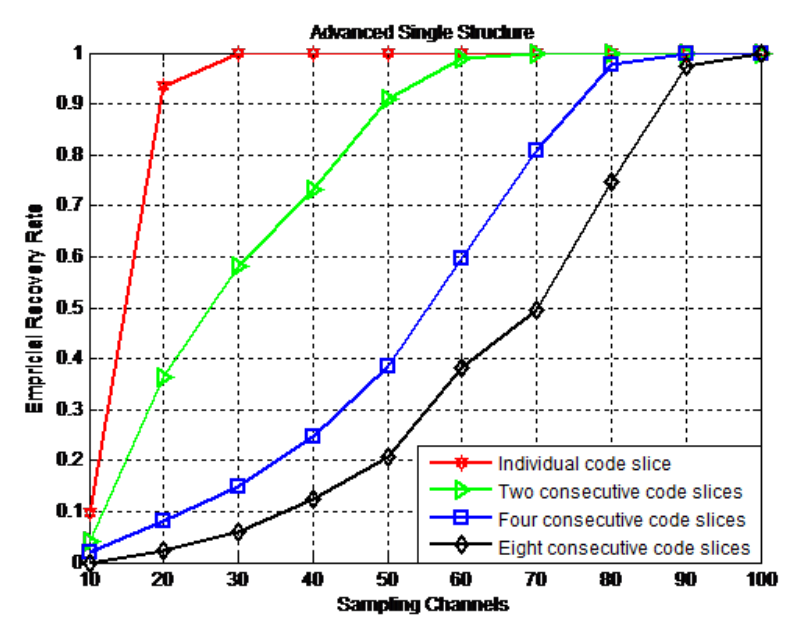

Figure 16 Percentage of correct support recovery in ADS, when SNR=0dB.

transform, the equivalent sub-Nyquist sampling values of multiple sampling channels are obtained by adding a frequency shifting module. The proposed structure can greatly reduce the number of hardware components, providing theoretical support for the sampling equipment towards miniaturized and intelligent. The single-channel structure employs only one mixing function, getting rid of the design of the mixing function. Moreover, the proposed ARED algorithm no longer requires the a priori information of signal sparsity, which can better meet the requirements of the actual complex electromagnetic environment. Simulation results show that the ADS system can achieve the similar performance as MWC with more simplified system structure, and even higher than MWC in some cases.

\section{Abbreviations}

MWC: modulated wideband converter; ARED: sdaptive residual energy detection; 5G: Fifth Generation; loT: Internet of Things; WSS: wideband spectrum sensing; CS: compressed sensin; AIC: analog to information converter; MCS: multicoset sampling; OMP: orthogonal matching pursuit; SMV: Single Measurement Vector; MMV: Multiple Measurement Vectors; ADS: advanced sampling.

Competing interests

The authors declare that they have no competing interests.

\section{Funding}

Heilongjiang Postdoctoral Financial Assistance under Grant No. LBH-Z19168. 


\section{Acknowledgements}

This work was supported by Heilongjiang Postdoctoral Financial Assistance under Grant No. LBH-Z19168.

\section{Availability of data and materials}

Data sharing is not applicable to this article as no datasets were generated or analysed during the current study.

\section{Author details}

${ }^{1}$ Harbin University of Science and Technology, No.52 Xuefu Road, Harbin, China. ${ }^{2}$ Harbin Institute of Technology, No.2 Yikuang Street, Harbin, China.

\section{References}

1. Araniti, G., lera, A., Pizzi, S., Rinaldi, F.: Toward 6g non-terrestrial networks. IEEE Network, 1-8 (2021). doi:10.1109/MNET.011.2100191

2. Liu, S., Gao, Z., Wu, Y., Kwan Ng, D.W., Gao, X., Wong, K.-K., Chatzinotas, S., Ottersten, B.: Leo satellite constellations for $5 \mathrm{~g}$ and beyond: How will they reshape vertical domains? IEEE Communications Magazine 59(7), 30-36 (2021). doi:10.1109/MCOM.001.2001081

3. Centenaro, M., Costa, C.E., Granelli, F., Sacchi, C., Vangelista, L.: A survey on technologies, standards and open challenges in satellite iot. IEEE Communications Surveys Tutorials 23(3), 1693-1720 (2021). doi:10.1109/COMST.2021.3078433

4. Sun, H., Nallanathan, A., Wang, C.-X., Chen, Y.: Wideband spectrum sensing for cognitive radio networks: a survey. IEEE Wireless Communications 20(2), 74-81 (2013). doi:10.1109/MWC.2013.6507397

5. Ma, Y., Gao, Y., Cavallaro, A., Parini, C.G., Zhang, W., Liang, Y.-C.: Sparsity independent sub-nyquist rate wideband spectrum sensing on real-time tv white space. IEEE Transactions on Vehicular Technology 66(10), 8784-8794 (2017). doi:10.1109/TVT.2017.2694706

6. Tropp, J.A., Laska, J.N., Duarte, M.F., Romberg, J.K., Baraniuk, R.G.: Beyond nyquist: Efficient sampling of sparse bandlimited signals. IEEE Transactions on Information Theory 56(1), 520-544 (2010). doi:10.1109/TIT.2009.2034811

7. Mishali, M., Eldar, Y.C.: Blind multiband signal reconstruction: Compressed sensing for analog signals. IEEE Transactions on Signal Processing 57(3), 993-1009 (2009). doi:10.1109/TSP.2009.2012791

8. Mishali, M., Eldar, Y.C.: From theory to practice: Sub-nyquist sampling of sparse wideband analog signals. IEEE Journal of Selected Topics in Signal Processing 4(2), 375-391 (2010). doi:10.1109/JSTSP.2010.2042414

9. Gao, Y., Chen, Y., Ma, Y.: Sparse-bayesian-learning-based wideband spectrum sensing with simplified modulated wideband converter. IEEE Access 6, 6058-6070 (2018). doi:10.1109/ACCESS.2017.2778699

10. Peng, H., Liu, M., Chen, L., Liu, Y.: An improved recovery algorithm based on isd for multiband signals, 1-5 (2017). doi:10.1109/WCNC.2017.7925798

11. Qi, P., Li, Z., Li, H., Xiong, T.: Blind sub-nyquist spectrum sensing with modulated wideband converter. IEEE Transactions on Vehicular Technology 67(5), 4278-4288 (2018). doi:10.1109/TVT.2018.2794779

12. Zhu, J., Li, J., Li, J., Li, Z.: Relevant support recovery algorithm in modulated wideband converter. IET Communications 13(18), 2883-2888 (2019)

13. Vasavada, Y., Prakash, C.: Sub-nyquist spectrum sensing of sparse wideband signals using low-density measurement matrices. IEEE Transactions on Signal Processing 68, 3723-3737 (2020). doi:10.1109/TSP.2020.3000637 